\title{
Confirmatory analysis of a health state classification system for people living with dementia: a qualitative approach
}

\author{
Alyssa Welch' (D, Nguyen Kim-Huong ${ }^{2}$, John Quinn ${ }^{3}$, \\ Caroline Gregory ${ }^{4}$, Wendy Moyle ${ }^{5}$, Julie Ratcliffe ${ }^{6}$ and \\ Tracy Comans ${ }^{7}$
}

\begin{abstract}
Objectives: This paper aims to confirm the content validity of the domains identified during the development of the Alzheimer's disease - five dimensions (AD-5D) algorithm for the quality of life - Alzheimer's disease (QOL-AD) and to identify the rationale for stated quality of life preferences.

Methods: Focus groups were conducted to elicit the priorities for quality of life in dementia from three perspectives: the person with dementia; family caregivers; and the community. Participants were recruited through industry research partners (long-term care providers) based on knowledge of their experience with dementia. Three focus groups were conducted - one each in Brisbane, Sydney and Adelaide, Australia - between November 2016 and February 2017. Each focus group included participants providing a different perspective on dementia - people with dementia $(n=3)$, caregivers $(n=9)$ and general community members or relatives of residents of a long-term care facility $(n=10)$, although some groups contained one participant with a different perspective. The focus groups were used to validate the AD-5D domains and examine quality of life preferences across the three perspectives. Thematic analysis was used to identify the priorities underlying preference selection.

Results: All activities affecting the quality of life for people with dementia could be mapped to one of the five AD-5D domains: memory, mood, physical health, living situation and ability to do things for fun. The domains considered most important for quality of life differed between people with dementia, their caregivers and members of the community, with memory the least important domain for all three groups. The rationale for priorities also varied between groups. Conclusions: This study confirmed the content validity of the selection of the AD-5D domains and identified multiple differences in the reasons behind stated priorities for quality of life for people with dementia, their caregivers and community members.
\end{abstract}

\section{Keywords}

dementia, proxy rating, quality of life, quality of life - Alzheimer's disease

\footnotetext{
'Research Manager, Centre for Health Services Research, The University of Queensland, Princess Alexandra Hospital, Australia ${ }^{2}$ Research Fellow in Health Economics, Centre for Health Services Research, The University of Queensland, Princess Alexandra Hospital, Australia

${ }^{3}$ Consumer Representative, Australia

${ }^{4}$ Clinical Psychologist, Centre for Health Services Research, The University of Queensland, Princess Alexandra Hospital, Australia ${ }^{5}$ Professor, Menzies Health Institute Queensland, Griffith University, Australia
}

${ }^{6}$ Professor of Health Economics, College of Nursing and Health Sciences, Flinders University, Australia

${ }^{7}$ Associate Professor \& Dementia Research Leadership Fellow, Centre for Health Services Research, The University of Queensland, Princess Alexandra Hospital, Australia

Corresponding author:

Alyssa Welch, Centre for Health Services Research, Princess Alexandra Hospital, Woolloongabba, Queensland 4102, Australia.

Email: a.welchI@uq.edu.au 


\section{Introduction}

The total number of people with dementia worldwide is projected to nearly double every 20 years, to 65.7 million in $2030^{1}$ and dementia is now the second leading cause of death for Australians. ${ }^{2}$ As the disorder is common in older populations, its incidence is expected to rise as the population ages.

The social and economic costs of dementia are significant. The cost of dementia to Australia in 2016 was estimated at AU\$14.25 billion ${ }^{3}$ and worldwide, in 2010, at US\$604 billion. ${ }^{4}$ Although there is currently no cure for dementia, a range of interventions (e.g. pharmacological, social care) have been trialled and implemented to relieve symptoms in an effort to improve both the quantity and quality of life for people living with dementia. Given the limited resources available to public and private bodies funding these interventions, economic evaluation of interventions is required to demonstrate their cost effectiveness.

The quality adjusted life year (QALY) is the key outcome measure utilized for the economic evaluation of healthcare interventions. Utility weights (a component of QALYs) are informed by the priorities assigned to the various domains of quality of life. Recent research indicates the importance of developing condition-specific (as opposed to generic) preferencebased measures that take into account the particular impacts on individuals' health and wellbeing of living with the condition. ${ }^{5,6}$ For people with dementia, a number of measures are frequently used to assess the condition-specific aspects of health-related quality of life (e.g. DEMQOL, Quality of Life - Alzheimer's Disease or QOL-AD). As originally developed, these measures have simple summative scoring systems and do not generate the utility weights required for determining QALYs for people with dementia for application within the framework of economic evaluation. ${ }^{7}$ More recently, preference-based scoring algorithms (the DEMQOL-U and DEMQOL-Proxy-U) have been developed for the DEMQOL to facilitate its application in this context. ${ }^{8}$

In 2016, Nguyen et al. ${ }^{9}$ conducted factor and Rasch analyses on the 13-item nursing home version of the QOL-AD that identified five core domains that were important for the health-related quality of life of people living with dementia (the Alzheimer's disease five dimensions or AD-5D): memory, mood, living situation, physical health and ability to do things for fun. ${ }^{9}$ This analysis laid the groundwork for a larger study to develop preference-based utility weights for the AD-5D to enable its use in economic evaluation of interventions for people with dementia.

This study presents the results of focus groups conducted to confirm the face validity of the AD-5D domains prior to undertaking discrete choice experiment (DCE) and best-worst scaling (BWS) activities online and via interview. ${ }^{10}$ Consistent with the design of the broader study, focus groups were conducted with people with dementia, caregivers and community members. Given the reliance on caregivers to provide proxy ratings of quality of life for people with dementia as the condition progresses, and taking into account established differences between self- and proxy-ratings of health-related quality of life, ${ }^{11-13}$ the reasons underlying quality of life priorities were compared between people with dementia, caregivers and community members. ${ }^{11-13}$ The aims of this study were to (1) confirm the content validity of the domains identified during the development of the AD-5D algorithm for the QOL$\mathrm{AD}$; and (2) to identify the rationale for stated quality of life preferences, including differences across stakeholder groups.

\section{Methods}

A qualitative approach was used to examine the content validity of the $\mathrm{AD}-5 \mathrm{D}$ domains. Comparative analysis was conducted on focus group outcomes to assess whether the AD-5D domains were comprehensive enough to capture the stated priorities of three different community groups, and identify whether early preferences were consistent with expectations. Examination of focus group transcripts aimed to identify whether the themes underlying stated preferences are consistent with those established in the literature. Ethical approval was granted from Griffith University Human Research and Ethics Committee (approval number 2016/626) and University of Queensland Human Research and Ethics Committee (approval number 2017001481). Written consent was received from all participants prior to the commencement of focus groups.

A total of three focus groups were conducted across Brisbane, Sydney and Adelaide, Australia between November 2016 and February 2017. Group 1 (the dementia reference group) contained people living with dementia in the community $(n=2)$ and a caregiver for a person living with dementia $(n=1)$. Group 2 (the caregiver group) contained caregivers and former caregivers for people living with dementia $(n=8)$ and one person living with dementia. Group 3 (the community group) contained former caregivers and relatives of residents of a long-term care (LTC) facility $(n=10)$. In all, 22 people participated in the focus groups. Each focus group was approximately $2 \mathrm{~h}$ in duration. Participants in Sydney and Adelaide were recruited through industry partners (aged care service providers). Participants in Brisbane were recruited through a local dementia support group. 
A combination of structured and unstructured methods were used to elicit quality of life priorities. Focus group participants were asked to independently and quietly answer the question "what are the everyday things and activities that contribute to or take away from your quality of life?' No specific examples were provided, participants were required to consider what this meant for them personally. Participants were given note paper to record their thoughts and were not limited in their selection of activities. All focus group participants were then asked to share their top two activities with the group, starting with one person and continuing around the group until no new activities were identified. This method allowed all participants to have a voice and avoided discussion being dominated by more vocal participants.

Psychometric analyses previously conducted to develop a dementia-specific health state classification system based on the QOL-AD ${ }^{9}$ identified five domains that could be used to develop QALYs for dementia: physical health, mood, memory, living situation and ability to do things for fun. These domains were explained and participants were asked to assign the identified activities to a domain. Acknowledging the interrelationships between domains, participants were given the option to duplicate activities if they thought they belonged to more than one domain. Participants were also asked to identify if they believed an activity did not belong to any particular domain.

Once activities had been assigned to domains, participants were given 10 'points' and asked to assign them to the most important contributors to their quality of life as a reflection of each activity's relative weighting. No limit was placed on the number of points that could be assigned to an individual activity.

\section{Analysis}

Thematic analysis was used to identify concepts and themes from transcribed digital audio recordings of focus groups. Transcriptions were reviewed for accuracy by two members of the research team (AW and CG). The framework method ${ }^{14}$ was used to analyse the transcripts, with the a priori framework developed based on the focus group structure and current literature on quality of life for people with dementia. Concepts and themes were coded individually by two researchers (AW and CG) in Microsoft Word and reviewed for consistency. Where conflicts arose in coding, a third researcher (TC) was consulted. Both reviewers examined interview transcripts to identify the influence of the facilitators on group responses, particularly checking for clarity of direction around freedom to map activities to any domain or no domain.
To accommodate the varying numbers of participants in each focus group, priority points were normalized across groups at the domain level by calculating the priority points assigned to items for each domain as a percentage of overall points allocated by the focus group. This enabled indicative comparisons of the importance assigned to each domain across the groups.

\section{Results and discussion}

Activities identified during the priority elicitation task and the 'points' or weightings assigned to them during the focus groups are presented in Table 1. Some activities, such as cognitive stimulation, family relationships, emotional support, and physical and financial independence were identified by all focus groups. Several activities were allocated to more than one domain. For instance, social interaction/engagement was allocated to the domains of mood, do things for fun and living situation; and sleep was allocated to all five domains. No activities were identified that could not be mapped to at least one of the five domains. Group 1 (the dementia group) identified several unique activities, such as the fear or stigma of dementia in the community, reclaiming identity and laughter.

Of the 18 activities prioritized by the reference group (group 1), only six were prioritized by the caregiver group (group 2) and four by the community group (group 3). Cognitive stimulation, support and encouragement, friendship and family were prioritized by all groups. This limited overlap in priority activities may be attributable to the smaller numbers in the dementia group, thereby limiting the opportunity for the same activities to be identified.

The normalized results of the prioritization task are presented in Figure 1. The percentage of priority points assigned to living situation was higher than any other domain for both the reference group $(40 \%)$ and the community group $(35 \%)$. The caregiver group assigned more priority points to the ability to do things for fun $(31 \%)$ than any other domain. Memory was assigned the fewest percentage of priority points by all three groups. Notably, the reference group (group 1) rated mood as considerably more important $(30 \%)$ than the other group groups (13\% for the caregiver group and $16 \%$ for the community group).

Analysis of the transcripts of each focus group identified several themes underlying activity choices.

\section{Focus group I - the person with dementia}

The perspective that participants in the group 1 brought to discussions of quality of life was generally a determination that a dementia diagnosis not be seen as the end of a person's ability to be themselves and live 


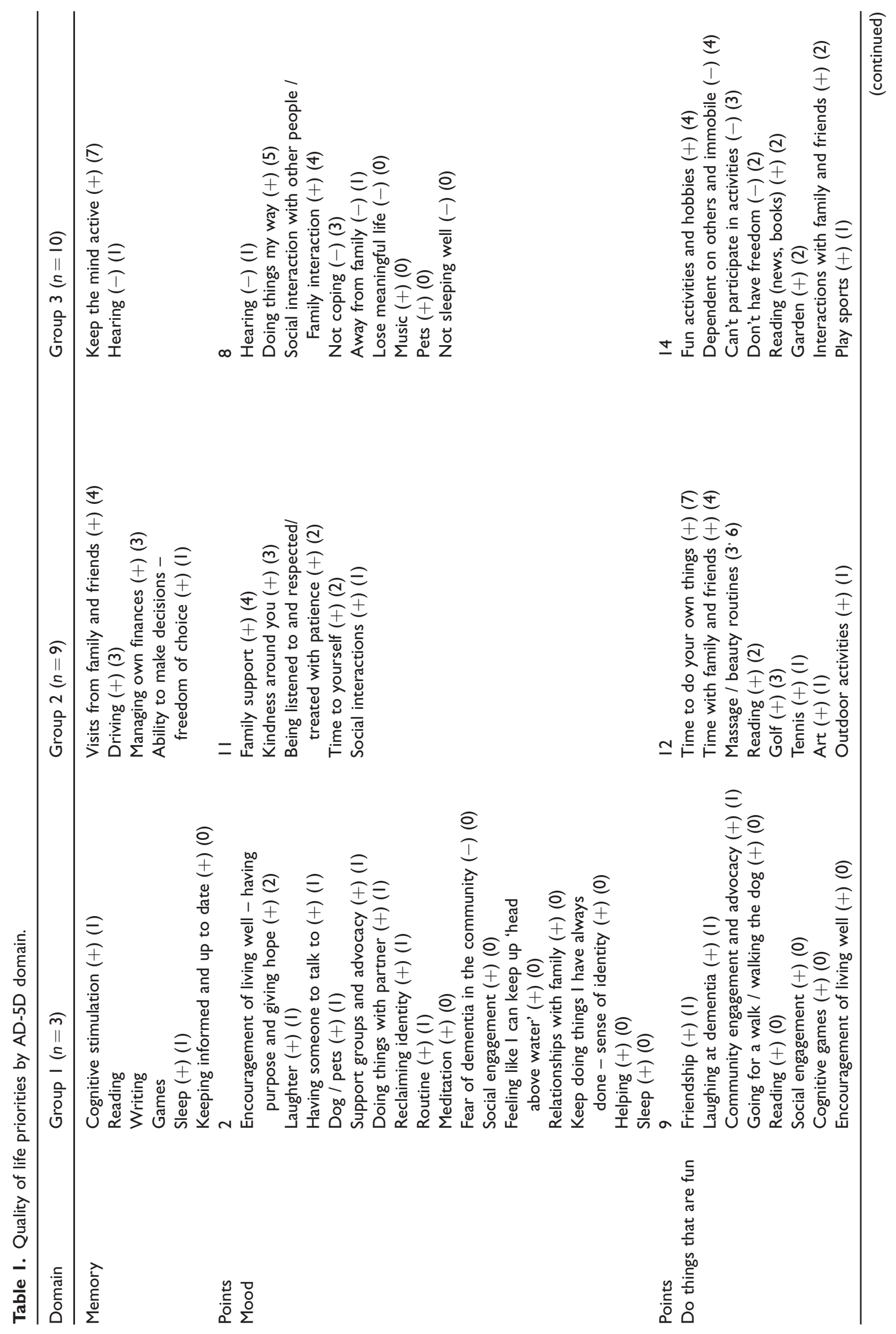




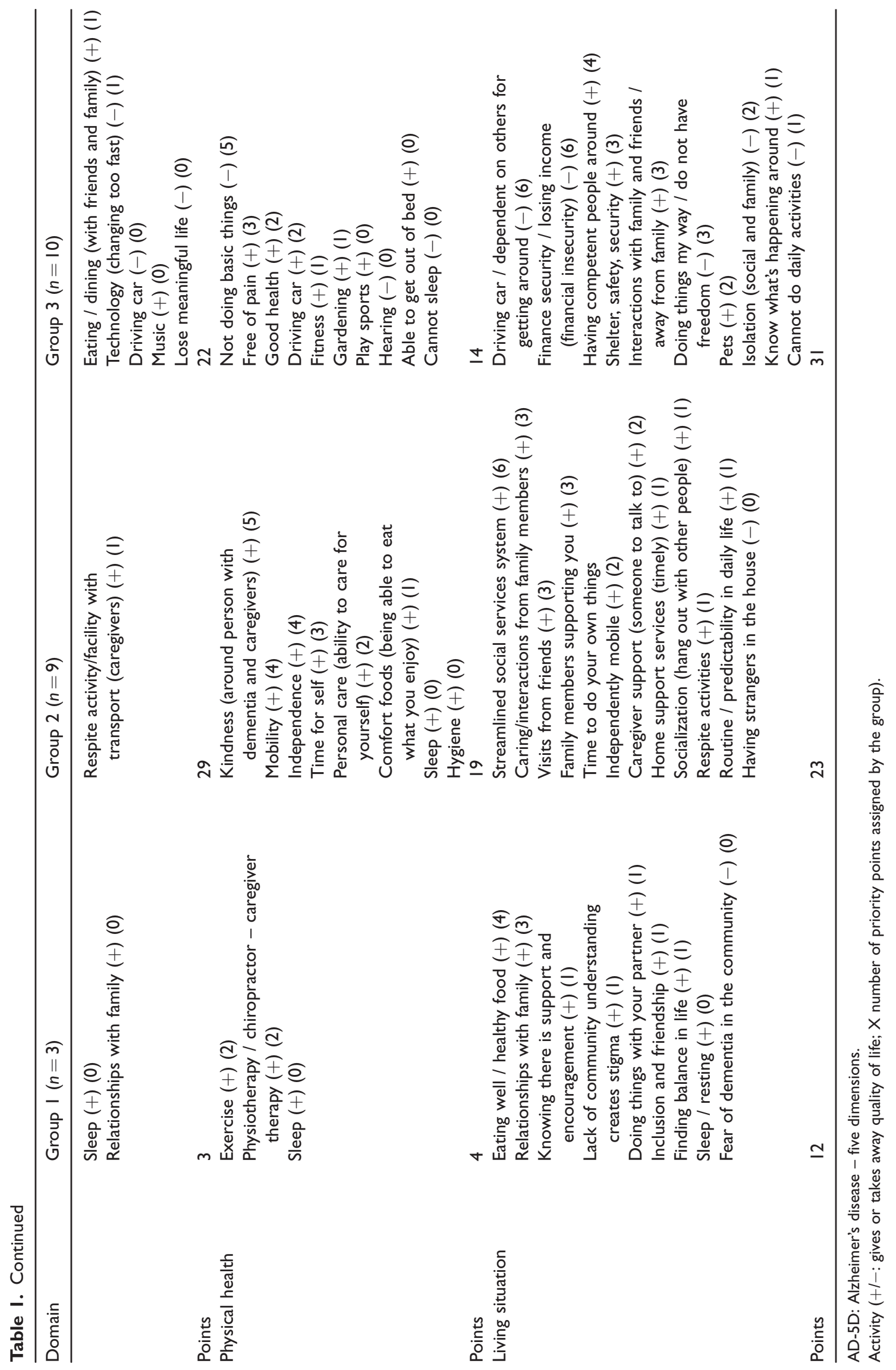




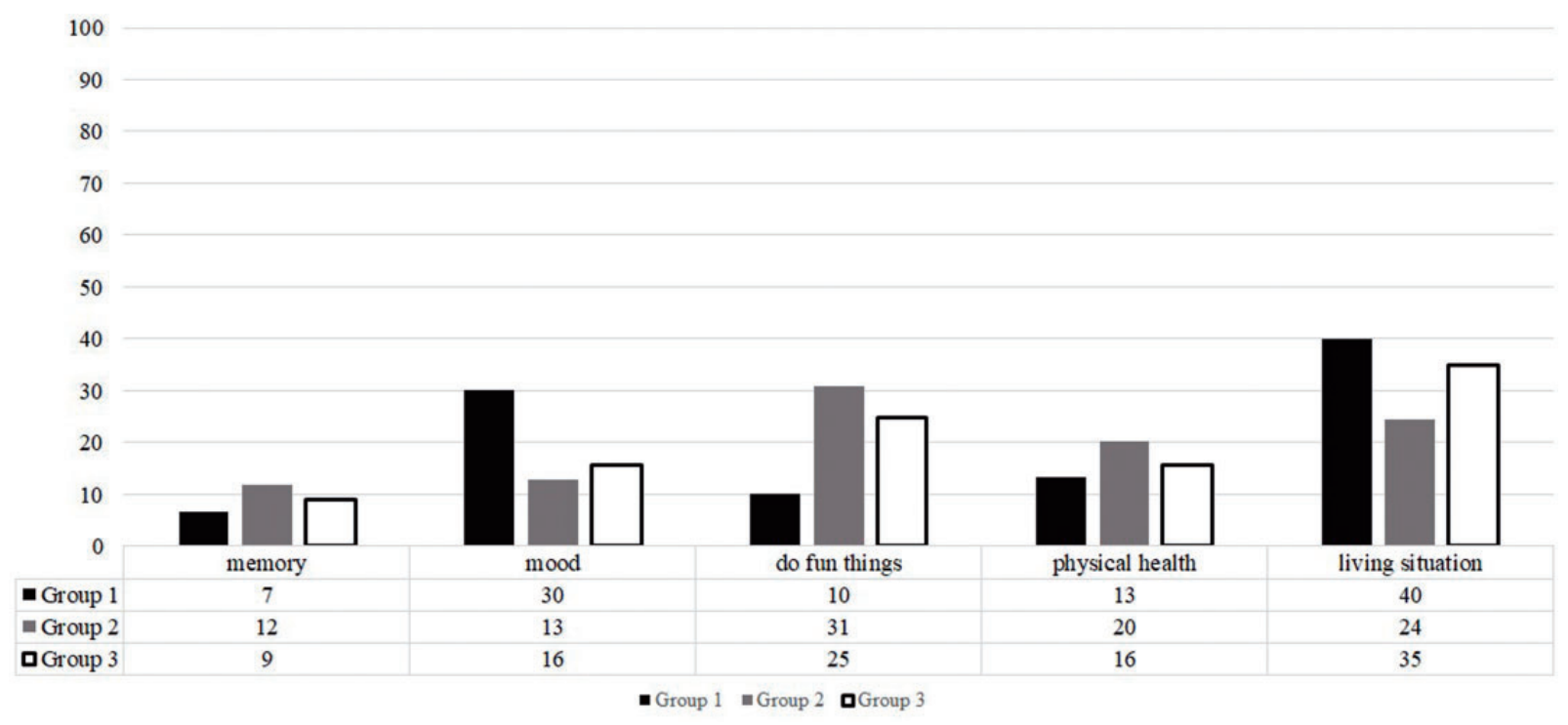

Figure I. Comparative weighting of priority points assigned by participants.

a fulfilling life. The empowerment and enablement of people with dementia to be able to maintain their identity through connection to what they can do, what makes them who they are, was seen as pivotal to a good quality of life. Contributing factors to quality of life included relationships, social engagement and support; having a sense of purpose and identity; maintaining wellbeing for continued enjoyment of life; and maintaining a sense of humour, optimism and positive attitude. The main detractor from quality of life was the stigma dementia participants believed was expressed in the community.

\section{Relationships, social engagement and support}

Being supported and encouraged by loved ones, sharing activities, sharing experiences through support groups and social engagement underlay many of the activities selected as contributors to quality of life. One person with dementia said "knowing that [caregiver] is there to encourage me, support me, to do the things I enjoy most' added to their quality of life. Another cited the feeling of inclusion that comes from support groups.

\section{Having a sense of purpose and retaining identity}

Having a sense of purpose, such as advocacy or keeping fit, created a sense of empowerment and provided opportunities to meet new friends and keep informed. The importance of retaining individual identity and allowing people to do the things they enjoy and are able to do also empowers individuals and returns a sense of purpose. This was demonstrated in an exemplar quotation: 'There's a mindset that the person with dementia has lost the cognitive ability to be able to interact.'

\section{Maintaining wellbeing for continued enjoyment of life}

Many of the activities identified (e.g. sleep, having a healthy diet and exercise) related to maintaining overall wellbeing, principally as an avenue for enabling participation in enjoyable activities. This was reflected in the statement of one participant 'I think everyone here, including myself, values the encouragement of living well.'

\section{Maintaining a sense of humour, optimism, positive attitude}

Having a sense of humour about dementia and the changes it brings to daily life helped both people with dementia and caregivers to cope with the challenges of dementia. One participant cited the importance of both the caregiver and the person with dementia 'Laughing at things that happen during the day because you've got dementia.' An optimistic attitude also created a sense of hope among participants that you could live well with dementia, engage in meaningful activities and pursue your interests.

\section{The stigma of dementia}

The stigma associated with dementia in the community frequently recurred as the underlying cause of detractors from quality of life. Community attitudes increased the impact of diagnosis, which could be compounded by childhood memories of other family members with dementia. One participant commented that 
diagnosis was 'a real blow to the system. And the reason for that is because of the stigma that's abounded for decades.' Upon their dementia diagnosis, another participant recalled memories of a family member being lost at a local shopping centre in their pyjamas. The perception of fear of dementia in the community and the reluctance of many community (and family) members to discuss dementia and its symptoms created a sense of isolation. For example, one participant described: 'My granddaughter asked me if I was going mad.'

There was also a sense that misconceptions of dementia prevented initiatives that may improve quality of life. For instance, 'there's a misconception that you can't enhance someone's life because you can't fix their memory.'

\section{General observations}

People with dementia participating in the focus group were initially reluctant to reveal their diagnosis and developed strategies to avoid detection of their symptoms. They stated that their reluctance was due to a fear of their family's reaction, fear of losing their job and fear of a change in the way they would be treated by others.

These factors are largely consistent with those established elsewhere, ${ }^{15-17}$ incorporating concepts such as relationships, agency in life and wellbeing. However, much of the existing quality of life literature focuses on people with dementia living in LTC facilities. The people with dementia in our focus groups were all living in the community, which may explain the importance of the perceived stigma attached to dementia among the general community. This perceived lack of understanding from general members of the community about what dementia is and the many different ways it presents was a cause of considerable distress among our community-dwelling participants.

\section{Focus group $\mathbf{2}$ - the caregiver}

The caregiver focus group was conducted to gain insight into caregiver perceptions of quality of life for people with dementia. These perceptions affect not only the quality of life of the person with dementia, but also the decisions caregivers make when providing proxy ratings of quality of life for the person with dementia. The caregivers in the 'caregiver' focus group, although prompted to discuss quality of life for the person with dementia, could not help but bring their own caregiving experiences to discussions. Many activities that improved quality of life for caregivers were ones that promoted the caregiver's sense of independence and 'otherness' from the caring experience. Caregivers also noted that independence and a sense of space were equally important for the person they cared for. Many participants also manifested grief for the loss of the life they had prior to diagnosis, and/or of the person with dementia as they moved further and further from the person they used to be. Contributors to quality of life included identity and sense of self; independence and space; and the joy of caring for a loved one. Detractors from quality of life for caregivers included a sense of loss and sacrifice; and changes to family and personal relationships.

\section{Identity and sense of self}

Many of the activities identified as priorities for improving quality of life (such as driving, mobility and reading) were selected because they enabled purposeful activity and a 'sense of self'. For instance, one caregiver stated, of the importance of mobility if you can't walk or drive, you can't do anything to help people.' One participant noted that the experience of caring for a person with dementia had altered their identity, by leading them to learn more and become an advocate for people with dementia 'I read books and anything I have found on dementia or dementia research, is because I've immersed myself into dementia research to try to identify an avenue where we can get better support for people with dementia and their carers.'

\section{Independence and space}

The need for independence and for time and space for yourself was a frequently recurring theme among participating caregivers. This theme drove many of the activity selections, such as massage, golf, tennis, art, outdoor activities and respite activities. One noted, however, that it was not just the caregiver who desired independence and quiet time, 'as much as I wanted time to myself and privacy and to just sit outside and do nothing, it's also what [the person with dementia] wanted and he would get very frustrated sometimes when we had caregivers coming over.' Freedom of choice was also an important aspect of independence for both caregivers and the person they cared for, for instance, 'being able to choose and have access to things that you had before that you like and comfort foods, that kind of thing.'

\section{Joy of caring for a loved one}

Some participants noted that they derived a lot of joy and satisfaction from caring for a loved one. Comments included, 'I developed a lot of joy out of looking after [the person with dementia]' and 'we had a 
wonderful marriage and I do believe I was born to be [my husband's] caregiver and I'm doing the best I can.'

\section{Sense of loss and sacrifice}

Throughout the focus group, caregivers returned to the themes of loss, grief and sacrifice as detractors from their quality of life. A loss of social interaction, family support and friendships were reflected in comments such as 'I've become more housebound where I was normally a very outgoing sort of person,' and 'your social life ... has changed. You know, people don't know what to say ... I wish they wouldn't ask me all the time because what am I going to say? He's not getting better ...' Grief for the loss of the life the caregiver and the person with dementia had prior to diagnosis and the sacrifices required when taking on full time care of a person with dementia were also raised, with one caregiver noting 'basically I don't have a life. I don't have my own life. I have [the person with dementia]'s life, but I don't have mine' and 'for 17 years [the person with dementia] was my life.'

\section{Changes to family and personal relationships}

The changes that caring for a person with dementia can bring to family and personal relationships was frequently raised by participants in the caregiver focus group. Some grieved for the loss of family relationships and support, evidenced through comments such as 'I've lost a lot of the time with my family,' and 'family and friends are only for good days, not to share the bad days.' Others were saddened by the changes brought to personal relationships, leading them to cross boundaries in their marriage, 'I thought when he got dementia, one thing. I'm not ... I'm never going to feed him, but I do.'

\section{General observations}

Other observations made by the participants in group 2 covered topics such as the importance of protecting the person with dementia's independence, the prohibitive cost of care arrangements not covered by government funding, and the importance of their own physical health. One participant commented 'I've got to keep myself fit because if something happens to me what is going to happen to [the person with dementia]?'

Although caregivers were asked to discuss aspects of quality of life for people with dementia, most discussion was about the experience of caring for a person with dementia. This inability to avoid discussing personal experiences is consistent with prior research ${ }^{15}$ and highlights the impact of the complex emotional, physical and social changes experienced by caregivers on the quality of life of the person with dementia.

\section{Focus group 3 - the community}

The discussion of quality of life among the community group (general community members and relatives of residents of a LTC facility) was focused on activities that brought enjoyment, with little insight into the reasons underlying their importance. The perspective that participants in the 'community' focus group brought to the discussion of contributors to and detractors from their quality of life was of not wanting to lose the things they currently enjoyed. Most comments were prefaced with 'I would miss ...' or 'I really enjoy doing ...' Emphasis was placed on the value of activities in their own daily lives right now. Contributors to quality of life were primarily those that increased comfort and the ability to do the things they love, such as health and strength. Participants generally expressed a reluctance towards a future with reduced capacity or a need to live in a nursing home, with one noting 'once you're locked up, you're locked up.'

The one caregiver who participated in the community group expressed similar sentiments to the caregiver group, including the personal and financial sacrifices required to provide full time care to a loved one.

\section{Discussion}

Results from the three focus groups confirmed that the important aspects of quality of life for people with dementia could be organized into five domains, thereby supporting the results of the previous factor analysis of the QOL-AD. ${ }^{9}$ This confirmatory analysis was important to allow a relatively simplified version of the QOL$\mathrm{AD}$ health states to be presented for valuation.

The differences between values assigned during proxy evaluations and self-evaluations are well established and the variations between people with dementia and caregivers noted in this paper were anticipated. The intention of the focus groups was not to demonstrate whether assessments evaluated with the AD-5D would be more valid or less susceptible to proxy issues than other instruments, simply to establish whether people with dementia were able to map all the activities they associate with quality of life to the AD-5D domains.

While all groups identified similar activities, differences were apparent in the relative value people placed on different aspects of their quality of life. The most important quality of life domain for people with dementia was their living situation, followed by mood and physical health. It will be interesting to see if analysis of the results of interviews with people with dementia and their caregivers confirms these findings.

The priorities of the caregiver group were more evenly distributed across the five domains, with living 
situation and doing things for fun weighted equally, closely followed by physical health. However, the caregiver preferences were elicited during group discussion, where shared experiences steered conversations towards the caring experience. DCE and BWS tasks will be completed with caregivers during one-on-one interviews, where each question will be prefaced by "what do you think the person with dementia would choose for this question?' to ensure participants attempt to respond with consideration to the person with dementia's preferences rather than their own. Planned analysis of DCE and BWS tasks with the general population will reveal whether the prioritization of these domains is consistent across different groups people with dementia; their caregivers; and the general community.

A limitation of this study was the lower than anticipated participation of people with dementia. Compared to the caregiver and community groups, only three people with dementia were able to attend the focus groups. Further, the person with dementia in the caregiver group made limited verbal contribution to discussions, with their caregiver speaking on their behalf. This was largely due to the symptoms of their dementia (slowness of speech) and an associated lack of confidence speaking in large groups. If forewarned, we would have made arrangements to interview this individual with their caregiver nearby, in order to differentiate their views from the views of the caregiver group. Consequently, these results should be viewed as indicative and should not be generalized to other people with dementia, caregivers or community members.

Another limitation of this study is that participants were asked to map quality of life priorities to predetermined domains, rather than arriving at the domains inductively. Although the facilitators regularly reminded participants that activities could be assigned to more than one domain and that items could be assigned to no domains (outside the scope of the AD-5D), adopting an inductive approach may have resulted in the development of different domains. However, this approach would not have fit the purpose of the focus groups, which was as a 'reality test' on the AD-5D domains prior to deriving preference-based utility weights.

This study has validated the AD-5D domains identified by Nguyen et al. ${ }^{9}$ with people with dementia, their caregivers and members of the community. The variations in priority domains and contributors to quality of life across the three groups have highlighted the importance of examining quality of life for people with dementia from multiple perspectives.

\section{Acknowledgement}

Data statement: due to the sensitive nature of the questions asked in this study, survey respondents were assured raw data would remain confidential and would not be shared.

\section{Declaration of conflicting interests}

The author(s) declared no potential conflicts of interest with respect to the research, authorship, and/or publication of this article.

\section{Funding}

The author(s) disclosed receipt of the following financial support for the research, authorship, and/or publication of this article: the National Health and Medical Research Council (NHMRC) Partnership Centre on Dealing with Cognitive and Related Functional Decline in Older People (grant number GNT9100000).

\section{ORCID iD}

Alyssa Welch (D) https://orcid.org/0000-0002-8873-9496

\section{References}

1. World Health Organisation. Dementia: a public health priority. Geneva: WHO, 2012.

2. In: Statistics ABo (ed) ABS causes of death, Australia, 2016. Australian Government, 2017.

3. Brown L, Hansnata E and Ha L. Economic cost of dementia in Australia: 2016 to 2056. Canberra: Dementia Australia, 2017.

4. Colucci L, Bosco M, Fasanaro AM, et al. Alzheimer's disease costs: what we know and what we should take into account. $J$ Alzheimers Dis 2014; 42: 1311-1324.

5. Hounsome N, Orrell M and Edwards RT. EQ-5D as a quality of life measure in people with dementia and their carers: evidence and key issues. Value Health 2011; 14: 390-399.

6. Neumann PJ. Health utilities in Alzheimer's disease and implications for cost-effectiveness analysis. PharmacoEconomics 2005; 23: 537-541.

7. Mulhern B, Smith SC, Rowen D, et al. Improving the measurement of QALYs in dementia: developing patientand carer-reported health state classification systems using Rasch analysis. Value Health 2012; 15: 323-333.

8. Rowen D, Mulhern B, Banerjee S, et al. Estimating preference-based single index measures for dementia using DEMQOL and DEMQOL-Proxy. Value Health 2012; 15: 346-356.

9. Nguyen K-H, Mulhern B, Kularatna S, et al. Developing a dementia-specific health state classification system for a new preference-based instrument AD-5D.(report). Health Qual Life Outcomes 2017; 15.

10. Comans TA, Nguyen K-H, Mulhern B, et al. Developing a dementia-specific preference-based quality of life measure (AD-5D) in Australia: a valuation study protocol. BMJ Open 2018; 8. 
11. Moyle W, Murfield JE, Griffiths SG, et al. Assessing quality of life of older people with dementia: a comparison of quantitative self-report and proxy accounts. $J A d v$ Nurs 2012; 68: 2237-2246.

12. Tay L, Chua KC, Chan M, et al. Differential perceptions of quality of life (QoL) in community-dwelling persons with mild-to-moderate dementia. Int Psychogeriatr 2014; 26: 1273-1282.

13. Huang HL, Chang MY, Tang JSH, et al. Determinants of the discrepancy in patient- and caregiver-rated quality of life for persons with dementia. J Clin Nurs 2009; 18: 3107-3118.

14. Gale N, Heath G, Cameron E, et al. Using the framework method for the analysis of qualitative data in multidisciplinary health research. BMC Med Res Methodol 2013; 13: 8 .
15. Smith SC, Murray J, Banerjee S, et al. What constitutes health-related quality of life in dementia? Development of a conceptual framework for people with dementia and their carers. Int $J$ Geriat Psychiatry 2005; 20: 889-895.

16. Beattie E, O'Reilly M, Moyle W, et al. Multiple perspectives on quality of life for residents with dementia in long term care facilities: protocol for a comprehensive Australian study. Int Psychogeriatr 2015; 27: 1739-1747.

17. Moyle W, Fetherstonhaugh D, Greben M, et al. Influencers on quality of life as reported by people living with dementia in long-term care: a descriptive exploratory approach. BMC Geriatr 2015; 15: 50. 